kituriger als den für das Atom, auf 18, einzuzeichnen. Gehen wir rom Kalium ritekwärts, so stolen wir auf Argon, das ron Natur 18 Eleltrotien hat - also so viele, wie die beiden betrachteten Elemente in ihrer Talenzbetatigung anrehmen - und das chemisch vollkommen inaktiv ist, also gar keine Neigung zeigt, von diesem Elektronengehalt abzugehen. Wieder um einen Schritt zuruek treffen wir das Cl, das elektrochemisch wieder einen ganz ausgesprochenen Cham rakter hat. Dieser Charakter ist aber entgegengesetzt dem der früher betrachteten Elemente, das Element ist negativ, in der Elektrolyse einwertiges Anion, trägt dort also ein Elektron mehr, als seinem neutralen Zustande entspricht. Demnach ist der Pfeil, der dies Verhalten kennzeichnet, nunmehr aufwärts zu zeichnen und, da das Atom 17 Elektronen enthält, für das Ion wieder ein Gehalt von 18 Elektronen anzugeben. Gehen wir pun nochmals einen Schritt zurück, zum Schwefel, und ermitteln wir den Elektronengchalt des $\mathrm{S}^{-}$-Ions, so erhalten wir wiederum jo 18. Fassen wir zusammen, so findet sich, daß die Elemente start polaren Charatters, die ein Edelgas umgeben, in ihrer Elehtrovalenzbetäti. gung stots dit Elehtronenzahl dieses Edelgases erreichen.

13. Ehe wir die Bedeutung dieses Ergebnisses fuir die Theorie der Valenzkräfte untersuohen, orientieren wir uns noch darüber, wie weit es sich ausdehnen läßt. Daß die übrigen im periodischen System jeweils in der Nähe eines Edelgases stehenden Elemente sich ebenso verhalten, wie die hier betrachtete Gruppe, erkennen wir ohne weiteres daraus, dab gerade hier die Elemente analoger Stellung. etwa die Alkalien, in der Elektrovalenz vollig übereinstimmen. Ebenso wie für die Elemente $\mathrm{nm}$ Argon. gilt der Satz also etwa für die von Sauerstoff bis Magnesium, die den Zustand des Neons anstreben, indem sie jeweils einen Gehalt von 10 annehmen. Hier deutet sich mun schon an einem geläufigen Beispiel an, daB das Gesetz noch weiter auszudehnen ist; das auf $\mathrm{Mg}$ folgende 13. Element $\mathrm{Al}$ ist als Kation dreiwertig, verliert also drei Elektronen, geht ebenfalis auf die Zahl des Neons zurück. Indes wird die Beobachtung dieses Falles schon durch HyArolyse selwieriger gemacht, und beim folgenden Eloment, Si, hat die Bildung warnehmbarer Menar? freier elementarer Kationen wiederum aufgehört.

Währead so die unmittelbare Beobachtbarkeit d'r Elektrovalenz untergeht, setzen die maximalen Hauptvalenz-Wertigkeitsstufen das gesetzmäBige Ansteigen von Element zu Element, das bei den Alkalimetallen einsetzt und mit der Elektrovalenz ithereinstimmt, hier bekanntlich noch weiter fort; fïr diese kommt etwa als ganze Reihe folgendes zustande:

die Halogenide: $\mathrm{NaCl} \quad \mathrm{MgCl}_{2} \mathrm{AlCl}_{3} \mathrm{SiCl}_{4} \mathrm{PCl}_{5} \mathrm{SF}_{6}$

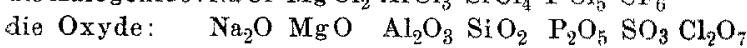

Wir nehmen nun an, dal alle Glieder dieser in sich zusammenhängenden Reihe gleicher Art sind, dal also die polare Konstitution, die sich in den ersten Gliedern durch Ionenbildung klar veruat, ihnen allen zukommt - eine Annahme, dic uns freilich dazu verpflichtet, später $z u$ begründen, warum die höherwertigen Mitglieder in Wasser weniger und weniger als freie Ionen auftreten. Es soll also jedes Halogenatom in diesen Verbindungen ein fremdes Elektron, jedes Sauerstoffatom zwei aufgenommen haben. Diese Elektronen muli jeweils der positive Teilnehmer der Verbindung hergegeben haben, ebenso wie die ersten Glieder der Reihe die Ionen $\mathrm{Na}+, \mathrm{Mg}+t$, $\mathrm{Al}+++$ abgeben, sollen die folgenden die Ionen Sit+t+, $\mathrm{P}+++++, \mathrm{S}+++++t$ und $\mathrm{Ol}+++++++$ in sich enthalten. Tragen wir diese Elektronenabgabe auf unserer Tafel ein, so zeigt sich, daB auch diese Elemente in ihrer Valenzfunktion vom Beispiel des Neons beherrscht werden.

Man erkennt ohne weiteres, daß man in ganz analoger Schlußweise die Reihe der negativen Ionenbildner vor dem Edelgas bis zum vierwertigen Kohlenstoff auszudehnen hat, indem man etwa von den ionenbildenden Wasserstoffverbindungen $\mathrm{HF}, \mathrm{H}_{2} \mathrm{O}$ zu den gesetzmäBig anschlieBenden $\mathrm{NH}_{3}, \mathrm{CH}_{4}$ fortgeht. Die von Neon beherrschte Reihe erstreckt sich demnach von $\mathrm{C}$ bis Ol, d. h. itber 12 Elemente. Analog beherrscht das Argon die maximalen Valenzfunktionen vom Si bis zum Mangan, das Krypton rom Germanium bis zum positiv 8-wertigen $\mathrm{Ru}\left(\mathrm{RuO}_{4}\right)$, d. h. sogar einen Bereich von 13 Stellen. Analoges gilt für Xenon und die Emanationen.

Die Zeichnung, die die maximalen Valenzfunktionen der Elemente bis zum Lanthan in der eben entwickelten Weise darstellt, läBt erkennen, daß neben den Elektronenzahlen der Edelgase noch andere als stabil hervortreten - sie geben zu den sogenannten "Nebenreihen" des periodischen Systems AnlaP -, dab indes allein die Edelgasformen so ausgezeichnet stabil sind, dab sie zur Aufnahme von Elektronen, d. h. zu negativer Funktion, AnlaB geben. Wir beschränken uns hier in unseren Beispielen auf diesen hervortretendsten Fall.

\section{(Schluf folgt.}

\section{Wanderheuschrecken und ihre Bekämpfung.}

Von Privatdozent Dr. K. Friederichs, Peanzenpathologe in Reichs-Kolonialdienst.

Das Heuschreckenproblem, wohl die größte Frage des Pflanzenschutzes, ist mehr un'd mehr aus dem primitiven Stadium der kleinen Mittel in das der planmäBigen Bekämpfung in großem Maßstabe eingetreten. Ist doch der einzelne fast machtlos gegen die oft unvermutet und von fern her kommenden Scharen, die ganze Landstriche verwüsten können. Nur ein organisiertes $\mathrm{Zu}$ sammenwirken, bei dem zumeist von Staats wegen 
dio Organisierung und selbst die praktische Durchführung stattinden muB, kann zum Ziele futhren. Kürzlich ist eine umfangreiche Monographie ${ }^{1}$ erschienen, welche auf Grund der in der Türkei während des Weltkrieges gemachten Erfahrungen die Plage und ihre Bekämpfung erörtert. Herausgeber ist der Regierungsrat Dr. Bücher, landwirtschaftlicher Referent im Reichskolonialdienst, dem eine Reihe von Mitarbeitern zur Seite standen. Auf Grund dieser Monographie und einiger anderer Literatur möge hier oine kurze Ubersicht uber die Heuschreckenplage und die Mittel zu ihrer Bekämpfung gegeben werden.

In Anatolien und Syrien handelt es sich um $z w e i$ verderbliche Arten. Die eine ist dis ägyptische Wanderheuschrecke, Schistocerca peregrina Oliv., die in manchen Jahren in ungeheuren geflügelten Scharen aus der Richtung von Ägypten her einwandert. Beheimatet ist sie nicht in der Türkei; zwar gelangen die Schwärme in dem Einfallsgebiet zur Fortpflanzung, aber nur eine, höchstens zwei Generationen entwickeln sich im Lande, später verschwinden diese Heuschrecken ganz wieder, da die Lebensverhältnisse dort fur vie nicht geeignet sind. Bücher sagt, es wäre von grober Wichtigkeit für die Türkei, wenn in len Ländern, von wo die wandernden Scharen kommen, d. h. im Sudan und in Oberägypten, eine Bekamplung stattünde; denn dort werde heute noch wenig oder gar nichts dagegen getan, trotz größter Schäden. Wenn darin ein Wandel eintritt, so wird die von dieser Art ausgehende Gefahr für Palästina und Syrien ron selbst beseitigt sein.

Stauronotus maroccanus Thunb. dagegen, die marolihanische Wanderheuschrecke, ist in der Türkei einheimisch. Sie ist auch in Nordafrika und Südeuropa weit verbreitet. Bücher gibt Einblicke in die Geschichte ihres Auftretens in Anatolien und Syrien and kommt zu dem Schlusse, dab sie daselbst seit langem einheimisch, daß das zentralo Westanatolien zu den permanenten Vexbreitungrgebieten za rechnen, das Zentrum des Vorkommens aber in den ausgedehnten Steppengebieten $z u$ beiden Seiten des mittleren Euphrat zu suchen ist. Die Biologie dieser Art ist in der genannten Monographie von Dr. W, La Baume ausführlich bebandelt. Seine Mittellungen beziehen sich auf die Lebensweise in Kleinasien. Tedes Weibchen legt nur einmal Eier ab und stirbt dann bald (andere Arten legen wiederholt Eier ab); es entsteht also in jedem Jahre nur eine Generation. da die Entwicklung sich lange

1) Monographien zur angewaudten Entomologie (Beibefte zur Zeitschr. f. angewandte Entomologie) Nr. 3. Die Heuschreckenplage thad ihre Bekämpfung. Unter Mitwirkung von Dr. $T$. Bawer, Dr. G. Breder mann, Dr. E. Fioliendey, Dr. W. La Baume und J. Loag herausgegeben von Dr, $H$. Bücher, Regierungsrat. 274 Seiten, 11 Karten, 33 Textabbildungen und $42 \mathrm{Ab}$ bildungen anf 20 Tafeln. Berlin 1918 (P. Parey). Preis 10 Ir. und $20 \%$ Teuerungsaufschlag. hinzieht, denn die Eier liegen 9 Monate in der Erde und liefern erst im nächsten Fruhjahre die neve Generation. Nach Reh (im Handbuch des Pflanzenschutzes gegen Tiere) können Heu* schreckeneier bei trockener Witterung auch melrere Jahre in der Erde ruhen, um erst auszuschlüpfen, wenn ein feuchteres Frühjahr eintritt

Die Gestalt der Eierpakete ist sehr mannigfaltig. Der Querdurchschnitt ist imner kreisförmig. Das obere Ende ist daran erkennbar, dal es gerade abgestutzt ist. Die Wandung besteht aus einer papierdünnen Schicht, an deren AuBenseite Erde, Sand und kleine Steinchen haften, die ihnen eine beträchtliche Festigkeit verleihen. Das oberste Drittel enthält keine Eier. sondern eine schaumige Masse, den "Schaumpfropf", der, wie die äußere Hülle, aus der ,Kintdruse" des Weibchens stammt. Das Paket enthält 30-35 Eier, die die ganze Eierzahl eines Weibchens sind.

Das Ausschlüpfen allex Embryonea, ihr Autsteigen zur Erdoberfläche findet gleichzeitjz, vielmehr bei allen unmittelbar nacheinander stat. Die Tierchen können dabei ihre Beine noch nicht gebrauchen, sondern sie schieben sich mit dem ganzen Körper nach oben. Zum Gebrauch ihrre GliedmaBen gelangt die Larve erst durch die sog. Embryonalhäutung, einen Vorgang, über den La Baume unsere Kenntnisse in erwünschtel Weise vermehrt. Vosseler sprach noch von der Hülle, welche den aufsteigenden Embryo, allen Teilen seines Körpers dicht anliegend, umgibt, als rom "Amnion". Es handelt sich aber vm eine echte Häutung, die ,Abstreifung einer echten, vom Integument abgesonderten und nicht als Eihaut entstandenen Cuticula" (La. Baume).

Die Lalve macht weitere fünf Häutungen durch, bis sie zur Imago oder zum Vollkerf wird. Schon im zweiten Stadium treten am Rücken kleine lappenartige Fortsätze, die Anlagen der beiden Flügelpaare, hervor. Auch die äußeren Genitalorgane der beiden Geschlechter sind schon in diesem Stadium deutlich verschieden. Im dritten Stadium lassen die Flügelanlagen berejts Andeutungen der Aderung erkennen. Im vierten haben sie an den Wurzeln eine gelenkartige Einschnürung bekommen, und während sie vorher nach unten gerichtet waren, liegen sie jetzt nach oben za auf dem Rücken. Im fünften lassen sie die Adern sehr deutlich erkennen und haben sivh stark vergröbert. Die nächste Häutung ergibt das geflügelte, erwachsene Tier, dessen Färbang zu dieser Zeit La Baume mit ,braunrosa" bezeichnet. Später ändert sich die Färbung; die alten Tiere sind , leuchtend weilgelb". Die Dauer jedes Larvenstadiums und damit die Dauer der ganzen postembryonalen Entwicklung ist Fränderlich; an einem Eiablageplatz bei Smyrna wurde sie auf 43 Tage festgestellt.

Es wurde schon gesagt, daB der Zeitpunkt des. Ausschlüpfens in hohem MaBe von klimatischen Faktoren abhängt; im übrigen aber sind sie im 
Paret in der Erde wohlyebrigen gegen Sommenintze oder Frost, Trockenheit oder Niederschläge. Weit abhängiger fom Klima sind die Larven und ueflügelten Heuschrecken. Freilkh ist das Klima 'hnen im westlichen Kleinasien sehr suinstig. 7war im März nnd noch im April können sie unter Kälte und Regen leiden, aber ihre ganze ubrige Entwicklung, einschließlich der Reifung ler Geschlechtsprodukte, fält in die Trockenzeit. Bevor aber die größte Sommerdurre eintrits, in jer die Vegetation verdorrt, ist thr Lebenskreis'aui vollendet. Tritt nun freilich eine ungewöhliche Witterung ein, was aber in Kleinasien $\mathrm{zn}$ ben Seltenheiten gehört, so wird sie den Heuschrecken verderblich. Insbesondere Iange lauerade Nässe schafft die Bedingungen für Infektionkkrarkheiten. Davon später.

Die gewöhnliche Fortbewegung dex Larven jst las Lanfen; nur zur Utberwindung von Hindernissen, zur Flucht und - zuletzt aber nicht am wenigsten - beim Marsch dient dazu der Sprung. Ceflugelte bewegen sich auf dem Erdboden fast nur durch Laufen. Thr Flugvermögen ist nach Ifo Baume von der Windrichtung unabhängig. Dagegen ist die Geschwindigkeit des Hhegens freilich ron Wind und Wetter abuangig, so auch lie Geschwindigkeit des Wanderns der Larven, - lie bei heißer Witterung besonders gro $B$ ist, im iibrigen aber abhängt ron dem Alter dex Larven ind rom Zustand der Vegetation. Nicht selten werden sie rom llunger zur Eile angetrieben. Gemessen werden kann die Geschwindigkeit eines Luges schwer: doch kounte $h_{a}$ Baume einmal bei Larven des vierten Stadiums rormittags in ler Sonne auf spärlich bewachsenem Boden feststellen, daß sie $1 \mathrm{~m}$ in der Minnte hetrug.

Wiewohl nicht sofort nach dem Ausschlupfen aus dem Ei auf Nahrung angewiesen - selbst âtere Larven und erwachsene-Tiere können mehrere Tage ohne Nahrung am Leben bleiben beginnen die jungen Heuschrecken doch alsbald za fressen, und zwar im Prinzip jegliche Pflanze; jedoch treffen sie, wenn kein Mangel herrscht, immerhin eine Auswahl. Bevorzugt werden z. B. Gramineen, daher leider auch die Getreidearten, ferner die Prerdebohne (Vicia faba). Die Futterwicke dagegen pflegt verschmäht za werden. cbenso der kultivierte Mohn u. a. Die Giftpflanze Peganum harmala wurde, wie Bücher feststellte, abgefressen. obgleich dies den Tod der Tiere znr Folge hatte, dis in Menge tot dabeilagen. Allerdings bestand die ganze Pflanzendecke an jener Stelle fast ausschlieblich aus dieser Pflanze, die Heuschrecken hatten also keine Wahl. - In erster Linie wird alles dicht am Boden Befindliche abgefressen, in Getreidefeldern nehmen die Tiere sich nicht Zeit, auf die hohen Halme hinaulznsteigen, es sei denn, dab der Sohwarm ruht. Geflugelte Heuschrecken frellich halten sich zuerst an die Ahren.

Auch bei diescr Art zirpt das Männchen, und zwar, wie es die Weise der Aridier ist, indem die Innuswte der Minterschenkel an der Flugeldecken reibt; der Ton ist nicht sehr laut, ry ertönt, renn es warm ist, bis spät in die Nacht. Im allgemeinen Ausdruck sexueller Erregung und bestimmt, das Weibchen anzulocken. kann der Zirplaut doch aush Erregungen anderer Art ausdrücken, z. B. wenn mehrere Männchen vich wn eimen Leckerbissen streiten. Er wird dann kürer. kräftiger und schriller als sonst (wzeugt.

Die Reifung der Geschlechtsprodulate erfolgt beim Männchen schneller als beim Weibchen. Wahrscheinlich ist der Same schon unmittelbar wach der letzten Häutung reif, doch ist diese Erage noch nicht genügend geklärt. Beim junren Weibchen ist das Ovarium kaum so groB wie 'in Stecknadelknopf, und es vergehen, bis es ausgewachsen ist, vier Wochen. Dann aber werden alsbald die Eier abgelegt, und das Absterben des Tieres erfolgt, so daB also auch die Dauer des Lebens der geflügelten weiblichen. Stauronotusheuschrecke vier IVochen dauert. Die Becattung ist lange vor dem Ablegen der Eier erfolgt. Vosseler erblickt die Ursache des späten Reifens der Eierstöcke darin, dab die Larve die hierzu nötigen Reservestoffe selbst für ibre Entwicklung verbraucht und nicht aufspeichern kann. Die frische geflügelte Heuschrecke enthält fast gar kein Fett, der Fettkörper wird vielmehr erst während des Lebens als Imago hinreichend ausgebildet. - Die Männchen sterben nach der Begattung nicht sofort ab, sondern leben noch wochenlang, ihre Lebensdauer ist nicht kiuzer als die der Weibchen. Diese exfahren nach der Erablage nochmals eine Begattung! Der Begattungstrieb der Männchen, deren Zahl größer zu sein pllegt als die der Weibchen, wird von La Baume als äuBerst heftig geschildert, auch crwähnt, daß mehrfach Versuche zur Begattung von Weibchen anderer Arten beobachtet wurden (Caloptenus italicus und Pachytitus nigroiasciatus).

Wenn die Zeit der Eiablage da ist, tritt eine merkwürdige Veränderung des Geschmackes der Heuschrecken ein. Sie setzen sich jetzt gern anf Odland fest, wo um diese Jahreszeit schon alle Vegetation verdorrt zu sein pflegt. Hier fressen sie gerade die vollständig trockenen, strohigen Pflanzenteile, daneben ihre toten Artgenossen, Kuhmist und anderes, was sie sonst nicht annehmen würden, verschmähen đagegen grüne Pflanzentriebe, nehmen jetzt auch Wasser am Rande von Gewässern auf.

Das Gelände, wo sie gemeinschaftlich die Eier ablegen, wählt der ganze Schwarm aus, sei es. dal der Schwarm geschlossen ihn aufsucht oder daß die Weibchen sich daselbst aus der. Ungegend ansammeln. Da Odland und Brachland im Hochsommer von weitem an ihrer braunen Färbung erkennbar sind, so leitet die Heuschrecken vermutlich der Gesichtssinn. Der Boden wird mit Aem Hinterleibsende betastet, und Grabversuche 
werden gemacht; ist er ungeeignet, so ziehen die Heuschrecken weiter.

,Man bemerkt auf den Eiablageplätzen erstens zahlreiche einzelne Männchen, zweitens eierlegende Weibchen. um die mehrere Männchen radial angeordnet herumsitzen, drittens Ansamm!ungen von Männchen, die sich auf dem Rücken eines eierlegenden Weibchens miteinander streiLen, und viertens Paare in Kopulationsstellung, dapunter oft Weibchen mit zwei Männchen auf dem Rücken. So bietet der Eiablageplatz ein - unBerordentlich mannigfaches und belebtes Bild Jar." (La Baume.)

Nur fester, bindiger Boden ist für das Ableyen der Eier geeignet, weil das Muttertier sich dazu mit dem Hinterleib in den Boden einwühlen muß. Sand oder Humusboden würde dabei den Grabzangen des Abdomens nicht genügend Widerstand bieten, ein Eingraben in hinreichende Tiefe nicht möglich sein. Geeignete, Plätze sind in Kleinasien vor allem die Hügel und Vorberge. Die fortschreitende Entwaldung daselbst hat sehr viele den Hewschrecken günstige Plätze geschaffen und somit viel zum Anwachsen der Heu3i:hreckenplage beigetragen.

Das interessanteste Krapitel der Biologie der Teuschrecken sind sicherlich ihre Wanderzüge. Die klaren Ausführungen $L_{a}$ Baumes werfen viel licht daranf. Zustande kommt die Wanderung lurch drei Faktoren: Ein ansgesprochener Geselligkeitstricb macht sich schon in der ersten Teit des Larvenlebens bemerkbar. Schon am Ende dier ersten Woche ihres Lebens beginnen sie, sich zunächst einzeln, nicht in geschlossener Masse, aber alle in der gleichen Richtung, ron dom Eihlegeplat\% zn entfermen. Bald wird die sogebannte Front gebildet, die scharf abgesetzte, sich nowärts schiebende vordero Grenze des Larvenzuges, der hier sehr dicht, nach hinten zul dünner ist. Manche Beobachtungsfälle lassen ferner auf einen Nachahmungstrieb schlicBen. Findlich ist oft eino Massenpsyche unverkennbar, indem seelische Erregungen, etwa durch äubere Störungen reranlabt, sich deutlich innerhalb des Zuges fortpllanzen; die entstehende Bewnruhigung ergreift besonders an der Front nach und nach die ganze Breite, so dal von weitem das Bild einer am Iffer entlanglanfenden Welle entsteht. Die Waulerzüge können einen oder selbst viele Kilometer ireit sein.

Die schwierigste und wichtigste Frage ist offenbar, wodurch die Richtung des Wanderzuges regeben wird. Die Beobachtung hat ergeben, daß im Geibirge die Wanderung im allgemeinen bergab erfolgt. Dabei wurde mehrfach festgestellt, dah inohrere Züge, die an ein und demselben Orte "ntstanden waren, unter genau gleichen Bedin rungen nach entgegengesetzten Richtungen wanherten. Somit hatten Witterungseinflüsse keiner1ı: Einfluo auf die Richtung. Alle Züge streben hergab und rereinigen sich mehr und mehr zu immer crößeren Kolonnen. Vielleicht werden die
Eüpfer ,bei Beginn ihrer Wanderung instinktiv in diejenige Richtung gedrängt, in welcher sie ihren Marsch mit geringstem Kräfteaufwand ausführen können" (Bauer).

Wenn also in hügeligem Gelände das Gefälle die Pichtung der Wanderung allein bestimnt, so müBte man erwarten, dal in der Ebene die Wahl der einzuschlagenden Richtung ganz regellos erfolgt. Nach Bredemanns Beobachtungen war in den meilenweit ausgedehnten Ebenen Nordsyriens in der Tat keinerlei gemeinsame Richtung zu erkennen. In den Ebenen Anatoliens hatte es nach La Baume in den wenigen Beobachtungsfällen den Anschein, als ob die Wanderung hauptsächlich dahin ging, wo reichliche Vegetation vie] Nabrung gewährleistete.

Die einmal eingeschlagene Richtung aber wird in der Regel für längere Zeit, meistens sogar für die ganze Dauer des Larvenlebens, festgehalten, wenn auch nicht immer genau. Dies ist für die Bekämpfung sehr wichtig, wie sich bei Darstellung der Methoden zeigen wird. - In geschlossene Wälder dringen die wandernden Heusohrecken nicht ein; ein Bach oder Fluß ist ilnen kein unüberwindliches Hindernis. Wenn auch ron der Strömung hinweggetrieben, erreichen die meisten friher oder später das andere Tfer.

Dic gröBte Gesamtstrecke, die Büchers Kenntuis zufolge cin Larvenzug zurückgelegt hat. ist 5 Kilometer. Fickendey aber hat in sehr unfruchtbaren Gegenden Anatoliens als Gesamtstrecke bis zu 20 Kilometer ermittelt. Wenn das Gelände eine üppige Vegetation trägt, so daB die Hüpfer reichliche und gute Nahrung finden, sn sehreitet die Front täglich nur einige Meter weiter ror.

Tonn dann im Anfang der siebenten Woche die ersten Larven ihre Flügel erhalten und damit z1. orwachsenen Tieren geworden sind, bei denen nur die Geschlechtsorgane, wenigstens wenn es Weibchen sind, noch erst reifen mussen, dann verändern diese zunächst ihr Verhalten im Wanderzuge in keiner Weise. Sie wandern zu FuB wie bisher, nur gelegentlich einmal, um einer Crefahr zu entgelen, entfalten sie im Sprunge die Flugel, aber zum Fluge selbst fehlt es ihrer Flügelmuskulatur noch an kräftiger Ausbildung. Darin ândert sich zunächst auch dann nichts, wenn bereits alle Lasven mit Flügeln versehen sind, sondern die Wanderung wird laufend und springend fortgesetzt. 10-12 Tage nach dem Erscheinen der ersten Geflügelten fangen die Massen au, sich zu begatten. Am 15. Tage beobachtete La Baume den ersten Flag, zuaächst nur ein planloses, yersuchsweises Umherfliegen ejnzelner, das aber dann andere zur Nachahmung reranlaßte. Da abor die Schwärme sich immer aus Tieren verschiedenen Alters zusammensetzen. so dauern alle die aufeinander folgenden Lebensvorgänge, wie Reifung, Begattung, Eierlegen nsw. innerhalb des Schwarms längere Zeit an. 
Derjenige Schwarm, an dem La Baume die phänologischen Daten feststellte, vermischte sich um diese Zeit mit einem Schwarm bereits geflügelter Heuschrecken, wodurch die Beobachtung gestört wuxde. Doch ist festgestellt, daB der erstere Schwarm nach dem Auftreten der ersten Vollreife noch drei Wochen lang an seiner Stelle verblieb. Es ist anzunehmen, dab dieser Zeitraum stets nicht unter 14 Tagen beträgt, da die jüngeren Larven exst ebenfalls heranwachsen müssen. Wenn es danu soweit ist, so wandert der Schwarm zuerst wochenlang unstet umher, wobei die Ebenen bevorzugt werden, weil daselbst noch gemügend Nahrung zu finden ist, während Hügel und Berge bereits diur sind. Aus zahlreichen Beobachtungen geht hervor, dab diese Flüge vorwiegend dem Winde entgegen erfolgten, wobei der Zug aber nur langsam rorwäts kommt. Fläge auf weite Entfernung sind zweifellos nux mit dem Winde möglich.

Frühestens vier Wochen, nachdem die ersten Geflügelten aufgetreten waren, sind bei der Mehrzahl der Weibchen die Ovarien reif, und es beginnt das Suchen nach einem gemeinschaftliohen Platze zum Ablegen der Fier, und sicherlich ist dieses Streben jetzt die Triebkraft der Wanderungen.

Von der Frage nach den unmittelbaren Ursachen des Zustandekommens einer Wanderung und nach den die Richtung gebenden Falstoren ist zu unterscheiden diejenige nach den inneren Ursachen und der Entstehung des Wandertriebes. La Baume betont das Hypothetische seiner AuBerungen darüber und sagt, daß die Abbängigkeit ron äuBeren Einflüssen noch bei weitem nicht genügend untersucht sei; es sei ein. Ding der Unmöglichkeit für ihn als einzelnen gewesen, im Laufe eines Jahres die gesamte Biologie vollständig zu erforschen, worin man ihm gewib Recht geben muB. Er hat aber erstaunlich Vieles und Genaues im Laufe dieses einen Jahres festgestellt, wie Ref. hinzusetzen möchte.

Die hypothetische Auffassung der Entstehung Jes Wandertriebes ist folgende: Der Bervegrrund für die Wanderung ist der Hunger. Vereinigung zu gröBeren Gesellschaften ist vorausgegangen. Diese aber hat notwendig die Wanderung zur Folge, weil, wo viele Tiere gleicher Art zusammen fressen, die Nahrung bald knapp werden muß. Bei Herdentieren reicht nun der Instinkt des Hungers allein nicht aus, das Bestehen der Art zu sichern. Denn, während ein einzelnes Tier nach erfolgtex Sätigung das Wiederauftreten des Hungers abwarten kann, muB eine grobe Gesellschaft von Tieren rechtzeitig vorsorgen, daB für alle rechtzeitig wieder genügend Nahruñg zu finden sei. ,Unter dem Zwange solcher durch das Zusammenleben in großen Massen bedingter Verhältnisse hat sich als ein besonderex Instinkt der Wandertrieb herausgebildet, dex die Heusclirecken veranlabt, die Frabstelle stets eher zu verlassen, als die absolute Notwendigkeit hierzu eintritt; nnd dey die Erhaltung des Tebens nud damit die der Art trotz den dureh die Massenbildung hervorgerufenen Schwierigkeiten der Ernährung sichert" Es handelt sich also um "eine durch Massenaniäufung bedingte Anderung des Triebas zur Nahrungssuche".

Im letzten Abschnitt des Lebens der Henschrecke aber tritt ein anderes Motix in don Vordergund: Die Sorge um die Nachkommenschaft. Dann werden die besten Futterpiätze verlassen und dürre, öde Strecken aufgesucht, wo whrend des oft wochenlang dauernden Anfenthaltes daselbst nur ganz rerdorrte Vegetation zur Verfügung steht - weil dicht und üppig bewachsener Boden zum Eingraben der Eier niaht geeignet wäre. Die Vererbung mag dann aus dioser Not eine Tugend gemacht, die gesamte Physiologie der Heuschrecken in diesem Abschuitt ihres Lebens verändert haben, dergestalt. dab ihre Geschmaaksrichtung sich rerändert, und sie aufhören, Grün $z$ fressen, und damit auch. unmittelbar Schaden anzurichten.

Die Teuschrecken haben viele natürtiche Feinde - aber, während bei manchen audere. Schadinsekten es bereits möglich gewesen ist, diesen biologischen Faktor zu ihrer Bekämpfung nutzbar zu machen, ist das bei Heuschrecken bisher kaum der Fall gewesen, und auch die Erfahrungen in der Turkei haben darin keine Anderung gebracht. D'Herelles Coccobacillus aridiorum zwar ist, das kann heute als gesichert gelten, pathogen für Heuschrecken. und kann, unter bestimmten Voraussetzungen, mit Erfolg zur Verbreitung einer Seuche unter thnen angewendet werden; aber diese Voraussetzungen sind solche, daB man es nicht in der Hand hat, sie herbeizuftihren, wie feuchle Witterung und einc infolge derselben eintretende - Schwächung der Heuschrecken, die sie für Krankheiten empfängIich macht. Uberdies kann dieses Vexfahren schwerlich den Vergleich ausbalten mit den sicher wirkenden meehanischen Mitteln und kann sie keineswegs ersetzen ${ }^{1}$ ). Übrigens - ist gerade Stauronotus maroccanus natïrlichen. spontanen Erkxankungen sehr ausgesetzt, wenn ungünstige Lebensverhältnisse eintreten, und solche können dann eine vernichtende Wirkung ausüben, ohnt dab es dazu eines Anstoßes von seiten des Menschen bedürfte ${ }^{2}$.

Wichtige tierische Feinde sind manche Vögel. Wrähon, die einen Eiablageplatz entdeckt haben, durchsuchen mit ihrem kräftigen Schnabel den Exdboden nach Eierpaketen. Den Schwärmen

1) Vgl. die Veröffentlichungen von Béguet, Musso und Sergent (1915), von Velu wnd Bouin (1915) und ron Velu (1916) im Bull. Soe. Path. Exot. (Paris).

2) So auch bei anderen Heuschrecken. 7. B. bra richtet Rutgers (in Meded, Labor. Plantenziekten. Batavia 1916) von einer durch den Pilz Metarrhicium anisoplita im Gefolge starker Regenfalle im Januar 1916 in Mitteljava bei den Heuschrecken der Spezie: Cyrthacanthacris nigricornis eingetretenen Seuche, durch welche dieselben innerhalb weniger Wochen fast vollig verschwanden. 
adel Larvenzügen folgen nicht selten Ansammlungen von Vögeln mehrerer Arten, die man als Eeuschreckenvögel zusammenfabt. In Anatolien fortgesetzte Bemrubiguog derselben, Lärm mit Blechgeräten usw.

Dia Fier werden am besten duruh Emoflügu sind es: Schwalben, Falken, Staare, Rosenstaare und Störche.

Parasitisclie und räuberische Insekten stellen ros allem den Eiern nach. Abgesehen von Käfern aus der Familie der Meloiden, dio zuweilen in die Eierpakete eindeingen und halb parasitisch darin leben, sind es Fliegen, Bombyliden, won denen aber bisher nur eine Art, Calostoma fasciI. enne Maca., genauer beobachtet worden ist. Ihre Eier setzt sie vermutlich einzeln auf den Erdboden $a b$, und die daraus hervorgehenden Larven bohren sich in die Erde ein, wo sie auBer von anderer, unbekannter Nabrung auch von den Stauronotusm ciern leben. In manchen Gegenden sind 30 bis $50 \%$ der Eierpakete von solchen Eliegenmaden befallen. Auch eine Muscide lebt in Eierpaketen, und zwar als Parasit, eine andere als solcher in den Heuschrecken selbst, d. h, die Maden entwickeln sich im Körper der lebenden Heuschrecke. Sie verlassen die Heuschrecke, deren Tod dann eintritt, zur Verwandlung in die Puppe und Eliege zwar nicht früher, als deren Leben normalerweise abgelaufen wäre, aber die von ihnen befallenen Heuschrecken, moist Weibchen, iroduzieren keine Eier.

Es war so viel über die Biologie zu berichten, $A a B$ es notwendig ist, die Methoden zur mechauischen Bekämpfung lurz darzustellen; der interessierte Leser wird darüber in dem Bücherschen Werke selbst nachlesen. Gassner hat 1909 iher die Bekämptung in Lruguay ${ }^{1}$ ) Mitteilungen remacht. Dort handelt es sich um Schwärme, lie ron auben her, ror allem aus dem nöndlichen Argentinien, eindringen; in Uruguay selbst können sie sich nicht auf die Dauer halten, da las Klima dort zu kalt für sie ist. Wiewohl die fliegenden Henechrecken, die, roladora", oft in wolkenartigen Sohwärmen kommen, so ist der Fchaden, den diese anrichten, doch im allgemeinen nicht sehr grob. Den Hauptschaden richten vielmehr exst später die im Laode selbst entstehenden Nachkommen an. Die Bekämpfung pichtet sich daselbst wie überall:

1. gegen die nukommenden fliegenden Schwärme,

2. gegen die abgelegten Eier,

3. gegen die Hüpfer und

4. gegen die zweite ,yoladora".

Gegen die voladora geht man anf nicht zu großen Flächen mit Erfolg vor, indem man in len ersten Morgenstunden, wenn die Heuschrecken noch klamm ron der Nachtkühle fast unbeweglich in IIaufen am Boden und sonstwo sitzen, dieselben einfach totschlägt. Im übrigen sucht ein jeder sein eigenes Feld durch Verm schenchen der Heischrecken zu schützen durch

1) G. Gassner, Heuschreckeneinfälle und ihre Bekämpfung in Uruguny. "Suid- und Mittel-Amerika", Halbmonatsschrift, Berlin, Hermann Paetel (1909). des damit belegten Geländes vernichtet. Aber es gibt riele Strecken, wo dies aus wirtschaftlichen Güuden nicht durchgefuhrt werden kann. Die Scharen der Hüper werden in Truguay zuweist durch Verbrennen abgetötet, indem man Stroh darüber wirtt, es mit Petroleum übergiebt und entzundet, Auch chemische Mittel, verseifte Petroleumlösungen oder Kreolinlösungen, kommen wenigstens gegen junge Hüpfer in Anwendung

Ortschaften schützen sich oft gegen die heranrückenden Scharen, indem die $z u$ sohützendex Grundstücke mit nicdrigen Wellblechzäunen um. stellt werden. Vox den Zäunen werden in Abständen Grubèn ansebracht, in welche die daran entlang wandernden Massen hineintallen; oft werden sie auch hineingetrieben. - Die Henschreckenbekämpfung ist in Truguay. wie hente in vielen Ländern, statlich organisiert.

Auch in der Türkei beruhte der bedeutende Erfolg der Bekämpfung auf der Schaffung einer vielverzweigten Organisation, durch welche dic Bevölkerung im Interesse der Sicherstellung der Ernährung mehr und mehr zu intensiver Behämpfungsarbeit herangezogen wurde. Die dabes gewonnenen Erfahrungen wurden rom den deatschen Sachverständigen zur Tervollkommnung tey Methoden benutzt.

$\mathrm{Zu}$ wirksamer Bekampfung der Heuschrecken ist die Anwendung verschiedener Mittel nebeneinander erforderlich; die Auswahl der Mittel im cinzelnen Falle hängt von den Umständen, inshesondere der Beschaffenheit des Landes und natürlich ron dem Stadium, in dem sich die Schädlinge befinden, ab.

Die Eier werden im einfachsten Falle mit der Hand gesammelt, nachdem der Boden autgehackt worden ist. ZweckmäBiger ist das Hacken der Brutstätten ohne nachfolgendes Sammeln. Die Eierpakete werden dabei teils verletzt oder zerquetscht, teils gehen sie durch Witterungseinflïsse und die sich ansammelnden Vögel zugrunde: Wo es angängig ist, kann an Stelle des Fackens das Umpflügen treten. Die Wirkung auf die Eier ist die gleiche wie beim Hacken, außerdem sehlemmt auf dem gepflügten Felde ler Regen Bodenteilchen zusammen, so daß eine harte Kruste entsteht, welche die ausschlüpfenden Larven im Frühjahr (das Pflügen muB im Herbst oder Winter erfolgen) nicht zu durchbrechen vermögen. Eine solches Feld wird überdies im nächsten Jahre von den Heuschrecken nicht als Brutplatz benutzt, da die rauhe Furche sie abschreckt.

Gegen die Hüpfer richten sich erstens die alten, in der Turkei einheimischen Treibeverfalren. Entweder wird ein Kesseltreiben oder cin Spiraltreiben veranstaltet. In beiden Fälen hat man das Bestreben, die Larven auf einen engen Raum zusammenzutreiben, um sie dort $z u$ 


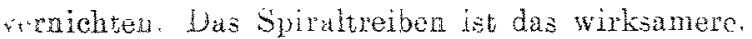
it die Heuschreaken gern seitwärts auswetchen, sine Neigung, der das Spiraltreiben Rechoung srägt. Die Vernichtung der Tiere erfolgt vielfach durch Zertrampeln oder Totschlagen mit beiaubten Zweigen; dabei retten sich viele Hiipfer lurch die Flucht; auch ist es zu zeitraubend. Besser ist das Eineintreiben in Gruben; auf steinigem Gelände aber treibt man die Larven statt lessen auf Tücher, Tscharschafs genanat, die $3 \mathrm{~m}$ breit und $5 \mathrm{~m}$ lang sind. An einom Sehlitz un Mittelpunkt ist ein Sack angenäht. Die Tscharschafs sind ron dunkler Farbe, da die Heuschrecken sich auf ein weiBes Tuch nur mit rroBem Widerstreben treiben lassea.

$\mathrm{Zu}$ diesem Verfahren gehören reiohlich viel A woitskräfte; auch können sie nur gegen kleineve Heuschreckenzüge zur Anwendung kommen, sind auch nach Bücher meist nur unvollkommen in der Wirkung. Es scheint in der Türkei bisher nicht üblich geweser $z u$ sein, die Eüpfer gagen sogen. cyprische Wände zu treiben, eine 1862 von dem cyprisohen Grundbesitzer A. Mattei erEundene Methode, die später in anderen Ländern ubernommen und verbessert worden ist. Dabei wird den Hüpfern eine Wand aus Leinwand im Winkel entgegengestellt, an der entlang Gruben ungebracht sind, in die sie hineingetrieben werden. Auf den Philippinen werden, wie ich aus Rehs Handbuch entnehme, die Zuckerrohrfelder mit auf den Kopf gestellten Bananen umgeben, an denen die Hüpfer entlang wandern, worauf sie dann in die an den Ecken befindlichen Gruben hineinfallen.

In der Türkei ist unter Büchers Leitung das Frinzip, den Heuschrecken vermittelst einer Wand den Weg abzuschneiden, in erster Linie und in sehr großem Maßstabe angewendet worden, ind zwar in der Gestalt der Zinkmethode. Zinkwände, Wellblechplatten, sind seit, langem in vielen Ländern bei dèr Heuschreckenbekämpfung 1n Gebrauch, aber während man anderswo die Heuschrecken dagegen treibt, ist das Verfahren jetzt in der Türkei vervollkommnet, ja man kann sagen eine nene Methode geschaffen worden in der Weise, daß man die Zinkwand selbsttätig wirken läBt. Man errichtet quer zu der Richtung, in der ein Zug wandernder Hüpfer heranzieht, eine aus ca. $30 \mathrm{~cm}$ hohen Zinkplatten bestehende Wand und verlegt ihnen so den Weg, denn überspringen können sie diese Wand nicht, auch nicht daran hochklettern. Sie wenden sich daher nach der Seite und wandern an der Wand entlang. An dieser sind aber in gewissen $Z$ wischenräumen Fallgruben angebracht, in die sie hineinfallen. Jegliches Treiben und Scheuchen ist dabei überflüssig oder gar schädlich, und während bei dem Treibverfahren wohi immer ein mehr oder weniger beträchtlicher Teil der Tiere entwischen wird, können sie bei sachgemäBer Anwendung der Zinkmethode fast restlos eingefangen. werden. Es bedar aber datu einer gröberen $Z a h l$ von Arbeitern, die won gesehultem Personal beaufsichtigt werden und die, schreibt Bücher, ,wie der Soldat im Felde, einem Führer auf das Wort gehorehen müssen". Auch ist es naturlich nicht mit zwei oder drei Zinkplatten getan. Das Personal wurde in für die Vernichtung von Heuschreckenzügen normaler Größe ausreichende ,Kolonnen" eingeteilt, und die von einer Kolonne benötigten Gerätschaften bildeten einen "Zinkapparat". Dazu gehören 1500 Platten, jede von 2 m Länge. Alle technischen Einzelheiten nebst Modifikationen, die unter bestimmten Verhältnissen nötig werden, sind in Büchers Monographie angegeben.

Auch mit chemischen Mitteln wurde vorgegangen. Als Kontaktgift hat eine 2-prozentige Seifenlösung gedient, wenn es sich um Hüpfer des ersten Stadiums handelte, und diese konnten damit getötet werden; aber von der Anwendung wird abgeraten, da zur vollständigen Wirkung jedes einzelne Tier von der Lösung getroffen werden mul, die mit Druckspritzen oder Gießkannen über den $\mathrm{Zug}$ vèrteilt wird. Da es nicht möglich ist, alle zu treffen, retten sich viele. Von den innerlich wirkenden Giften wird am meisten emptohlen "Urania", eine von der Chemischen Fabrik in Schweinfurt hergestellte verbesserte Form des Schweinfurter, Grins, dem gegenüber es vielerlei Vorzuge besitzt, die hauptsächlich auf seiner Unlöslichkeit bewuhen. Es wird entweder auf die dem Wanderzug voraussichtlich zur Nahrung dienenden Pflanzen gespritzt, also vor dem wandernden Zuge darauf gebracht und ist besonders nützlich, wo eine dichte, ziemlich lückenlose Vegetation den Boden bedeckt. Die andere Art der Anwendung besteht in dem Auslegen von Giftködern. Als Köder kann z. B. Luzerne - eine Lieblingsspeise der Heuschrecken - dienen, die in eine 1mproz. Aufschwemmung ron Urania getaucht und zwischen den Heuschrecken ausgestreut wird. Diese kämpfen förmlich um dieses Futter. Andere Lockspeisen sind Kleie, Mist; Häcksel, Sägemehl und Treber. Der Erfolg ist um so sicherer, je älter und hungriger die Tiere sind. Während das Spritzen in dichter Vegetation zu empfehlen ist, kommen für das Ködern naturgemäB Plätze mit spärlicherer Vegetation in Betracht. Dex Erfolg kann besonders bei älteren, hungrigen Zügen ein vollständiger bei einmaliger Anwendung sein. Bücher gibt mancherlei praktische Winke. Die Giftverfahren haben gegenüber dem Treibeverfahren den Vorzug, dab weniger Arbeiter dazu benötigt werden. Andererseits sind sio kostspieliger.

Auch wenn die Heuschrecken bereits geflügelt sind, kann Gift gegen sie angewendet werden, besonders, wenn sie in bebaute Felder bereits eingefallen sind, empfiehlt sich dies. In der Bücherschen Monographie ist von der Bekämpfung Geflügelter nicht viel die Rede, da er es eben ganz überwiegend mit Hüpfern zu: tun hatte. Truthuhinerherden können, besonders in junge Mais- 
Ealler eingetrieben, gute Dienste leisten. Damit kommen wir nochmals zu den biologischen Veriahren, nämlich insoweit sich Bücher vom prakischen Standpunkt dazu äußert. Auch er hebt un Wert des Waldes für eine dauemde Beseitisung der Heuschreckenplage hervor. Da sie in dichte Wälder nicht eindringen, so wäre ein Waldgürtel ein sicherer Schutz für die Kulturebenen. Dazu wäre er eine Fleimstätte einer den Feuschrecken feindlichen Tierwelt. Den Heushreoken widrige und Giftpflanzen wurden schon oben erwähnt. Zu den Pflanzen; die die Heushrecken nicht gern fressen, gehört die Kartoffel, äberdies macht das Abweiden eines Teils des Krautes nicht allzuviel aus. Insofern wäre iie Kartoffelkultur zu fördern. Beim Getreide "mptiehlt sich der Anbau stark begrannter Sorten. Die Grannen werden zuerst gefressen, oft lann die Körner verschont. Besonders von Beleutung aber ist die Härte der Körner, die ihnen Schutz gewähren kann, so bei einer als ,Kandem har" bezeichneten Weizensorte in Syrien. - Es kann vorkommen, daB der Heuschreckenfraß nutzlich ist! Wenn die ,Akdari" genannte Sorm shum- (Hirse-) Art jung abgeweidet wird, so wird dadruch der Ertrag erhöht! Wenigstens behaupten die anatolischen. Bauern das. Auch anleres junge Getreide, das abgeweidet wird, erholt ich vollständig.

Bücher legt mit Recht großen Wert auf die statliche Organisation der Bekämpfung, die in allen Einzelheiten dargelegt wird. Ohne solche wäre jede Heuschreckenbekämpfung aussichtslos. Uber die erzielten Erfolge äuBert sich Bredemann, der die Bekämpfung in Nordsyrien und Nordinesopotamien leitete, daß daselbst gegen alle stadien der Heuschrecken gründlicher Erfolg zu verzeichnen war, und dab die Ernte völlig sicheryestellt wurde. Auch sei in groben Gelbieten die Ablegung von Eiern völlig verhindert worden, und demnach im folgenden Jahre keine Heuschreckenplage daselbst zu erwarten. Man weis aber, daß die Hauptquelle der heranflutenden Heuschreckenmassen die Steppe ist. Auch dort wird man ilnen, nach Bredemann, zu Leibe gehen können und müssen, und zwar mit der Zinkmethode.

Die Hohe des Schadens, der noch 1916 in Anatolien von den Heuschrecken angerichtet wurde, schätzt Bücher auf 100 Millionen Mark! Dis Abwehrtätigkeit beschränkte sich in diesem Jahre hauptsächlich auf den Schutz der Felder. 1917 konnte die Bekämpfung in vollem Umfange aufgenommen werden, und es wurde nach Bücher erreicht, dab, auch in den stärlrstbefallenen Distrikten nennenswerter Schaden verhütet werden konnte". Genaue Zahlen sind schwer beizubringen, doch, steht fest, dab sich in diesem Jahre (1917) der durch Heuschreckenfrab angerichtete Schaden durchschnittlich nicht über das Maß des natürlichen Verlustes durch Hagelschlag usw. erhebt"6.

Aus den in Massen eingesammelten und abge- lieferten Eiern konnte Professor Dr. Beckmann (Kaiser-Wihelm-Institut für Chemie in Dahlem) ein Fett ausziehen, dessen Verwendung als Speisefett möglich erschiene, doch ist die Gewinnung desselben praktisch untunlich. Verwendung als' Geflügelfutter wäre an Ort und Stelle möglich, weiter Transport lohnt sich nicht. U̇brigens sind, wie schon ausgefuhrt, andere Verfahren der Bekämpfung durch Einsammeln der Eierpakete vorzuziehen, die Frage der Verwertung derselben daher von geringer Bedeutung. Die getrockneten Heuschrecken sind, mit Ausnahme der jüngsten Stadien, ein Futter von hohem Nährwert, aber Hühner, an die man sie in Menge verfuttert, liefern minderwertige Fier. Das beste ist daher, den Inhalt der Fanggruben als Dünger auf die Felider zu bringen. -

Es sind sehr anerkennenswerte praktische und theoretische Leistungen, von denen die Monographie Kunde gibt. Wo immer die Heuschreoken künftig dem Menschen zu schaffen machen, wird man, um die Bekämptung auf die Höhe zu bringen, auf diese "Erfahrungen in der Ttirkei mit zurückgreifen müssen.

\section{Mitteilungen \\ aus verschiedenen Gebieten.}

Roland von Eötvös + Baron Roland won Eötvös, Ungarns gröBter Naturforscher, ist am 8. April in Budapest dahingeschieden. Als Sohn des Barons Josef v. Eätvös, des hervorragenden ungarischen Sehriftstellers und Politikers und ehemaligen Kultueministers, wurde er am 27. Juli 1848 zu Buda geboren. $\operatorname{Er}$ begaun seine akademischen Studien an der Pester Universitat und setzte sie in Heidelberg fort, wo er Kirchhoffs, Helmholtz' und Bunsens Vorlesungen besuchte; kurze Zeit verbrachte er auch in Königaberg, um Hranz Neumann zu hören. Nachdem er in Heidelberg the Doktorwürde erlangt hatte, habilitierte er sich an der Pester Universität als Privatdozent der Physik und wurde dort im Jahre 1872 zum ordentlichen Professor der theoretischen Physik und nach einigen Jahren auch der Experimentalphysik ernannt. Das neue physikalische Institut der Universitüt verdankt ihm seine Ausstattung, die, den Anforderungen der Zeit entsprechend, nicht nur zur Demonstration qualitativer Versuche, sondern auch zur Ausführung präziser Messungen' und exakter physikalischer Untersuchungen geeignet ist. Ganz besonders lag ihm die Vervollkommnung des Unterrichtswesens der Hochschule am Herzen, und mit deren vielseitiger förderung hat er wesentlich dazu beigetragen, daß Ungarn in die Reihe der Kulturvolker Europas eintreten konnte. Dasselbe bezwekten die weitreichenden kulturellen Institutionen, die er während der kurzen Dauer seines Amtes als $\mathrm{Mi}$ nister für Kultus und Unterricht ins Leben rief. An der Wirksamkeit der Ungarischen Akademie der Wissenschaften nahm er, mehrere Jahre als ihr Prisident, regen Anteil. Im Jahre 1891 gründete er die ungarische Gesellschaft für Mathematik und Physik, deren Vorsitzender er bis zu seinem Tode war. Als ungariseher Delegierter der Internationalen Erdmessungskommission nahm er auch an deren Arbeiten regen Anteil.

Kupfermerkblatt des Bureau of Standards. Das Bureau of Standards will von Zeit zu Zeit Merkblatter 